\title{
CANONICAL BACKWARD DIFFERENTIATION SCHEMES FOR SOLUTION OF NONLINEAR INITIAL VALUE PROBLEMS OF FIRST ORDER ORDINARY DIFFERENTIAL EQUATIONS
}

M. R. ODEKUNLE

(Received 12 March 2003; Revision accepted 29 September 2002)

\begin{abstract}
This paper describes a new nonlinear backward differentiation schemes for the numerical solution of nonlinear initial value problems of first order ordinary differential equations. The schemes are based on rational Interpolation obtained from canonical polynomials. They aro A-stable. The test problems show that they give better results than Euler backward method and trapozoidal method near a singular point.
\end{abstract}

KEYWORDS: backward differentiation scheme, collogation, initial value problems,

\section{INTRODUCTION}

Let us consider the initial value problem (ivp)

$$
y^{\prime}=f(x, y), \quad y\left(x_{0}\right)=y_{0}
$$

where $y=\left[y^{\prime}(x), y^{2}(x), \ldots, y^{s}(x)\right]^{\top}$ and $y_{0}$ is given. We assume that (1) satisfies the hypothesis of the existence and uniqueness theorem (Lambert, 1973: 2) in the integration interval $a \leq x \leq b$. Such ivps may either be stiff, oscillatory, singular or neither stiff nor singular nor oscillatory. It is usually desirable to have methods that possess stably efficient, accurate property to handle most if not all these cases (Hall and Watt, 1976:116). At the same time, cost must be considered. This is mostly determined by the number of function evaluations required at any integration step provided the scheme meets the desired minimum error tolerance.

Fatunla (1978) designed a variable order one-step scheme to cater for ivps whose solutions are osciliatory. Fatunla (1978 and 1988) and Lambert (1973) recommended the use of L-stable integrators for excessively stiff ivps. These and others are the motivator for the use of backward differentiation formulas (bdfs) in automatic codes for stiff differential systems. The use of bdf in the solutions of stiff ivps dated back to Curtis and Hirschfelder (1952). Since then, different automatic codes using bdf for stiff and non-stiff differential system have evolved. These include DIFSUB of Gear $(1969,1971)$ and its descendants such as GEAR (Hindmarsh, 1974), LSODE (Hindmarsh, 1980), DEBDF and others (Fatunla, 1988:27, 213-214).

In general, the known bdf can be described as (Lambert, 1973:12)

$$
y_{n, k}=h \beta_{k} f\left(x_{n, k}, v_{n, k}\right)+g
$$

where $g$ is a constant for the current step obtained as a linear combination of past values of $y$. When $k=1$, we have the implicit Euler or better known as Euler backward differentiation formula

$$
y_{n+1}=y_{n}+h f_{n+1}, \beta_{1}=1
$$

Problems involving singularities are very common in reality. Most of the available codes for such problems made use of equation (3). The purpose of this paper is to provide us with an alternative which we believe as shown in Table 2 should give a better result at a small extra cost due to the need to compute square root sign but basically, no extra function evaluation is needed when our proposed schemes is compared with equation (3) and trapezoidal method.

\section{CANONICAL POLYNOMIIALS}

Let the operator $\mathrm{T}$ be defined as (Taiwo and Onumanyi, 1991)

$$
T=\frac{d}{d x}+1
$$


and define

$$
T Q_{j}(x)=x^{j}
$$

Then,

$$
\begin{aligned}
& T x^{j}=j x^{j-1}+x^{i} \\
& T x^{i}=j T Q_{j-1}(x)+T Q_{j}(x) \\
& x^{i}=j Q_{j-1}(x)+Q_{j}(x)
\end{aligned}
$$

Therefore,

$$
Q_{i}(x)=x^{j}-j O_{j-1}(x), \quad j=0,1,2, \ldots
$$

From (4a),

$Q_{0}(x)=1, Q_{1}(x)=x-1, \quad Q_{2}(x)=x^{2}-2 x+2, \ldots$

These polynomials written in rational form shall be used to derive our bdfs

\section{DERIVATION OF THE METHOD}

Let $y(x)$ be the solution of $(1)$ and assume that

$$
y(x) \approx \bar{y}(x)=\frac{\sum_{i=0}^{n} a_{i} Q_{i}}{1+\sum_{j=0}^{n} b_{j} Q_{j}}
$$

where $a$ and $b$; are constants to be determined and $Q_{i}$ and $Q_{i}$ are as defined by (4a). Let us consider the case when $n=1$ only so that we can write (5) as

$$
\bar{y}(x)=\frac{a_{0} a_{0}+a_{1} a_{1}}{1+b_{0} a_{0}+b_{1} a_{1}}
$$

which on using (4b) becomes

$$
\bar{y}(x)=\frac{a_{0}+a_{1}(x-1)}{1+b_{0}+b_{1}(x-1)}
$$

Replacing $y$ in $(1)$ by $\tilde{y}$ we have,

$$
\overline{y^{\prime}}(x)=f(x, \bar{y}(x)), \quad \bar{y}\left(x_{0}\right)=\bar{y}_{0}, \quad x \in\left\lfloor x_{0}, x_{n}\right\rfloor
$$

Collocate ( 7 ) at $x=x_{n}$ and $x=x_{n+1}$ to obtain

$$
\overline{y^{\prime}}\left(x_{n}\right)=f\left(x_{n}, \bar{y}\left(x_{n}\right)\right)
$$

and

$$
\overline{y^{\prime}}\left(x_{n+1}\right)=f\left(x_{n, 1}, \bar{y}\left(x_{n+1}\right)\right)
$$

Using (6) in (8a) and (8b) we respectively obtain

$$
\begin{aligned}
& a_{1}\left(1+b_{0}\right)-a_{0} b_{1}=\left[1+b_{0}+b_{1}\left(x_{n}-1\right)\right]^{2} f_{n} \\
& a_{1}\left(1+b_{0}\right)-a_{0} b_{1}=\left[1+b_{0}+b_{1}\left(x_{n+1}-1\right)\right]^{2} f_{n+1}
\end{aligned}
$$


Equating the right hand sides of (9a) and (9b) we have

$$
\left(\frac{f_{n}}{f_{n+1}}\right)^{\frac{1}{2}}=\frac{1+b_{0}+b_{1}\left(x_{n+1}-1\right)}{1+b_{0}+b_{1}\left(x_{n}-1\right)}=F \text { (say) }
$$

so that

$$
b_{1}=\frac{\left(1+b_{0}\right)(f-1)}{x_{n+1}-1-\left(x_{n}-1\right) F}
$$

Flewrite the initial condition

$$
\vec{y}\left(x_{11}\right) \cdots y_{11}
$$

as

$$
v_{n}=\frac{a_{0}+a_{1}\left(x_{n}-1\right)}{1+b_{0}+b_{1}\left(x_{n}-1\right)}
$$

ive obtain

$$
a_{0}=\left(1+b_{0}\right) \bar{y}_{n}-\left(x_{n}-1\right)\left(a_{1}-b_{1} \bar{v}_{n}\right)
$$

Using $(12 a)$ in $(9 a)$ we obtain

$$
a_{1}=b_{1} y_{n}+\left[1+b_{0}+b_{1}\left(x_{n}-1\right)\right] f_{n}
$$

Substitute the value of $a_{1}$ as in (12b) in equation (12a) to obtain

$$
a_{0}=\left(1+b_{0}\right) \bar{y}_{n}-\left(x_{n}-1\right)\left[1+b_{0}+b_{1}\left(x_{n}-1\right)\right] f_{n}
$$

In (G), substitute the values of $a_{1}$ and $a_{0} a s$ in (12b) and (12c) respectively we have,

$$
y=\bar{y}_{n}+\left(x-x_{n}\right) f_{n} \frac{\left[1+b_{0}+b_{1}\left(x_{n}-1\right)\right]}{1+b_{0}+b_{1}(x-1)}
$$

Substitute $(11)$ in this equation we have

$$
y=\bar{y}_{n}+\left(x-x_{n}\right) f_{n} \cdot \frac{h}{\left(x_{n+1}-x\right)+\left(x-x_{n}\right) F}
$$

$\ln (13)$ let $x=x_{n}$, so that,

$$
y_{n+1}+v_{n}+h f_{n} \cdot \frac{1}{F}
$$

(a) If $F=1$ we have,

$$
\ddot{v}_{n+1}=\ddot{y}_{11}+h f_{n}
$$

the popular Euler formula, Euler (1913).

(b) If $F \neq 1$ and $F \neq 0$ then,

$$
\bar{v}_{n+1}=\bar{v}_{n}+h \frac{f_{n}}{F}
$$

and on using $(10)$ in this we have

$$
\bar{v}_{n+1}=\bar{v}_{n}-h\left(f_{n} f_{n+1}\right)^{\frac{1}{2}} \text { if } F<0
$$


or

$$
\bar{v}_{n+1}=\bar{y}_{n}+h\left(f_{n} f_{n+1}\right)^{\frac{1}{2}} \text { if } F>0
$$

These are our desired schemes.

\section{PROPERTIES OF THE SCHEMES}

We noticed that (15) represents non-linear implicit schemes. The determination of $y_{n+1}$ at $x_{n+1}$ with an assumed value $y_{n+1}^{(0)}$ requires predictor-corrector approach.

Theorem (Jain, 1987 )

Let $y_{n+1}^{(m)}$ be a sequence of approximations to $y_{n+1}$. If for all values of $y$ close to $y_{n+1}$ and including the

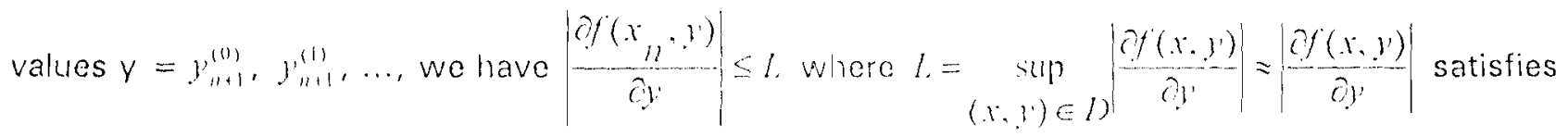
$|h L|<1$, then the sequence $\left\{y_{n+1}^{(\prime)}\right\}$ converges to $y_{1+1,1}^{\prime}, p \geq 0$.

This implies that for a converging sequence oi approximations, $F$ must be a decreasing function.

We next consider the concept of stability of the schemes with a view to access their suitability. Let us apply the schemes to Dahqquist (1963) stability scalar test.

Consider the ivp

$$
y^{\prime}=\wedge y, \quad y\left(x_{0}\right)=y_{0}
$$

From $(15 b)$ and $(16)$.

$$
\bar{v}_{n+1}=\bar{y}_{n}+h \lambda\left(\bar{v}_{n} \bar{v}_{n+1}\right)^{\frac{1}{2}}
$$

or

$$
\frac{y_{n}+1}{v_{n}}=1+h \lambda_{4}\left(\frac{y_{n}+1}{v_{n}}\right)^{\frac{1}{2}}
$$

Let

$$
p=\frac{\bar{y}_{n}+1}{\bar{y}_{n}}
$$

to obtain

$$
p^{2}-\left(h^{2} \lambda^{2}+2\right) p+1=0
$$

or

$$
p^{2}-\beta p+1=0
$$

where

$$
\beta=\lambda^{2} h^{2}+2>0, \quad \lambda \in ! i
$$

The same applies if we use (15a) and (16). The roots of the characteristics equation (17) are 


$$
p_{1}=\frac{\beta-\sqrt{\beta^{2}-4}}{2} \text { and } p_{2}=\frac{\beta+\sqrt{\beta^{2}-4}}{2} \text {. }
$$

If $\lambda h \rightarrow \infty, \beta \rightarrow \infty$ and the first root tends to zero while the second root becomes dominant. The relative error becomes unbounded oscillation and the computation degenerates into nonsense beyond a certain point. This shows that the schemes are A-stable only when $\lambda$ is positive (compared with Scheid (1989).

\section{ILLUSTRATION}

Investigation shall now be made about the performances of equations (15a) and (15b) using some problems. In both problems stated below, classical Runge - Kutta scheme of order 4 shall be used as the predictor. This is to reduce or eliminate errors due to the predictor as compared with that by the corrector so that the performance of each corrector can be easily seen. In each of the problems considered, the results shall be compared with that when linear backward Euler (equation (3)) is used being the corrector used by most of the existing codes. All iterations at each step shall be carried out once. That is, PEC mode [Lambert, 1973; 87] using the. classical Runge-Kutta scheme as the predictor shall be used throughout the iterations to give each scheme a fair trial and for easy comparison. No improvement strategy is introduced also. We have notwithstanding used double precision so that the error could be that of discretization only.

The choice of (15a) or (15b) in solving any problem from our computational experience illustrated using problems 1 and 2, depend on Lipschitz condition of the problem and $F$.

1. If as $F$ decreases $L$ also decrease, we use (15a) otherwise,

2. If as $F$ decreases $L$ increases, we use (15b).

Let us rewrite our schemes in simpler forms by replacing $\bar{y}_{n}$ by $y_{n}$ and $\bar{y}_{n, 1}$ by $y_{p+1}$ in Equations (15a) and (15b) to respectively obtain

$$
y_{n+1}=y_{n}-h\left(f_{n} f_{n+1}\right)^{\frac{1}{2}} \text { if } F<0
$$

or

$$
y_{n+1}=y_{n}+h\left(f_{n} f_{n+1}\right)^{\frac{1}{2}} \text { if } F>0
$$

These are the schemes that are now applied in solving the following examples.

Problem 1:

$$
y^{\prime}=-10(y-1)^{2}, \quad y(0)=2,0 \leq x \leq 1
$$

Theoretical Solution: $y=1+\frac{1}{110 x}, L=|-20(y-1)|$

Here, for a decreasing $F, L$ decays so we shall use equation (18a). The results are shown in Table 1.

Problem 2:

$$
y^{\prime}=1+y^{2}, y(0)=1,0 \leq x \leq 1
$$

Theoretical Solution: $y=\operatorname{lan}\left(x+\frac{\pi}{4}\right), L=|2 y|$

Type: Solution is oscillatory and has singularity at $x=\frac{\pi}{4}$.

For problems like this, as $F$ decreases, the Lipschitz condition increases and even exponentially near the singularity point (Fatunla, 1988: 26) so we shall use (1813). The results are shown in Table 2.

\section{DISCUSSION OF RESULTS}

In table 1, our results using (18a) to solve problem 1 is shown. Our results were compared with those obtained for the same problem using implicit Euler scheme equation (3). The absolute errors obtained using our scheme are in general smaller than those obtained using equation (3) although with an extra work of calculating square root but using the same number of function evaluations at each step. 
TABLE 1: The absolute errors for different values of $h$ for

$$
y^{\prime}=-10(y-1)^{2}, y(0)=2,0 \leq x \leq 1
$$

\begin{tabular}{|c|c|c|c|c|c|c|}
\hline \multirow[b]{2}{*}{$x$} & \multicolumn{2}{|c|}{$h=0.1$} & \multicolumn{2}{|c|}{$h=0.01}$. & \multicolumn{2}{|c|}{$h=0.0025$} \\
\hline & $Y_{n+1}=y_{n}+n f_{n+1}$ & $y_{n+1}=y_{n}-h\left(f_{n} f_{n+1}\right)^{05}$ & $y_{n+1}=y_{n}+h f_{n+1}$ & $y_{n+1}=y_{n}-h\left(f_{n} f_{n+1}\right)^{2}$ & $y_{n+1}=y_{n}+h f_{n+1}$ & $y_{n+1}=y_{n}-h\left(f_{n} f_{n+1}\right)^{0.5}$ \\
\hline 0.1 & $2.500000 \mathrm{E}-01$ & $3.725290 E-09$ & $1.885005 \mathrm{E}-02$ & $7.155910 \mathrm{E}-05$ & $4.431773 \mathrm{E}-03$ & $1.143664 \mathrm{E}-06$ \\
\hline 0.2 & $3.815104 \mathrm{E}-01$ & $4.166667 \mathrm{E}-02$ & $1.381415 \mathrm{E}-02$ & $1.694560 \mathrm{E}-04$ & $3.148109 \mathrm{E}-03$ & 1.135468E-05 \\
\hline 0.3 & $4.232922 \mathrm{E}-01$ & $3.710938 E-02$ & $9.847272 E-03$ & $1.506694 \mathrm{E}-04$ & $2.235886 \mathrm{E}-03$ & $9.771436 \mathrm{E}-06$ \\
\hline 0.4 & $4.249055 \mathrm{E}-01$ & $2.834456 \bar{E}-02$ & $7.301094 \mathrm{E}-03$ & $1.173043 \mathrm{E}-04$ & $1.660397 \mathrm{E}-03$ & $7.321834 \mathrm{E}-06$ \\
\hline 0.5 & $4.032961 \mathrm{E}-01$ & $2.144285 E-02$ & $5.624316 \mathrm{E}-03$ & $9.109742 E-05$ & $1.282545 \mathrm{E}-03$ & $5.455481 \mathrm{E}-06$ \\
\hline 0.6 & $3.670290 \mathrm{E}-01$ & $1.652354 \mathrm{E}-02$ & $4.470701 \mathrm{E}-03$ & 7.187833E-05 & $1.022894 \mathrm{E}-03$ & $4.369385 \mathrm{E}-06$ \\
\hline 0.7 & $3.224350 \mathrm{E}-01$ & $1.302708 \mathrm{E}-02$ & $3.644647 \mathrm{E}-03$ & $5.787797 \mathrm{E}-05$ & $8.359291 \mathrm{E}-04$ & $3.490597 \mathrm{E}-06$ \\
\hline 0.8 & $2.751978 \mathrm{E}-01$ & $1.049406 \mathrm{E}-02$ & $3.032799 \mathrm{E}-03$ & $4.744088 \mathrm{E}-05$ & $6.972287 \mathrm{E}-04$ & $2.953741 \mathrm{E}-06$ \\
\hline 09 & $2.301046 \mathrm{E}-01$ & $8.615647 \mathrm{E}-03$ & $2.566663 \mathrm{E}-03$ & $3.954530 \mathrm{E}-05$ & $5.915856 \mathrm{E}-04$ & $2.214909 E-05$ \\
\hline 1.0 & $1.819325 \mathrm{E}-01$ & $6.977004 \mathrm{E}-03$ & $2.202847 \mathrm{E}-03$ & $3.347614 \mathrm{E}-05$ & $5.087488 E-04$ & $1.632478 \mathrm{E}-06$ \\
\hline
\end{tabular}

TABLE 2: The absolute errors when $h=0.01$ for $y^{\prime}=1+y^{2}, y(0)=1$,

$0 \leq x \leq 0.80$ (singularity point is at $x \geq 7.857143 \mathrm{E}-01$ )

\begin{tabular}{|c|c|c|c|}
\hline$x$ & $y_{n+1}=y_{n}+h f_{n+1}$ & $Y_{n+1}=y_{n}+1 / 2 h\left(f_{n}+f_{n+1}\right)$ & $y_{n+1}=y_{n}+h\left(f_{n} f_{n+1}\right)^{\sigma 5}$ \\
\hline $1.000000 \mathrm{E}-02$ & 4.444040 E 04 & $6.463446 \mathrm{E}-04$ & $6.474174 \mathrm{E}-04$ \\
\hline $5.000000 \mathrm{E}-02$ & $4.377810 \mathrm{E}-04$ & $7.071051 \mathrm{E}-04$ & $7.131848 E-04$ \\
\hline $1.000000 \mathrm{E}-01$ & $1.907844 \mathrm{E}-03$ & $8.223141 E-04$ & $8.157311 E-04$ \\
\hline $1.500000 E-01$ & $3954204 \mathrm{E}-03$ & $9.202642 \mathrm{E}-04$ & $9.486360 E-04$ \\
\hline $2.000000 E-01$ & $6.849081 E-03$ & $1.075834 \mathrm{E}-03$ & $1.124590 \mathrm{E}-03$ \\
\hline $2.500000 \mathrm{E}-01$ & $1.102734 \mathrm{E}-02$ & $1.282931 \mathrm{E}-03$ & $1.363397 \mathrm{E}-03$ \\
\hline $3.000000 \mathrm{E}-01$ & $1.720910 E-02$ & $1.566365 \mathrm{E}-03$ & $1.697376 \mathrm{E}-03$ \\
\hline $3.500000 E-01$ & $2.627087 E-02$ & $2.515787 \vec{E}-03$ & $2.721780 \mathrm{E}-03$ \\
\hline $4.000000 E-01$ & $4.113123 E-02$ & $3.252536 \mathrm{E}-03$ & $3.599197 E-03$ \\
\hline $4.500000 \mathrm{E}-01$ & $6.594021 \mathrm{E}-02$ & $4.393991 \mathrm{E}-03$ & $4.996474 \mathrm{E}-03$ \\
\hline $5.000000 \mathrm{E}-01$ & $1.089636 \mathrm{E}-01$ & $8.094187 \mathrm{E}: 03$ & $9.156819 E-03$ \\
\hline $5.500000 E-01$ & $1.875876 \mathrm{E}-01$ & $1.588071 \mathrm{E}-02$ & $1.790107 \mathrm{E}-02$ \\
\hline $6.000000 E-01$ & $3.717247 E-01$ & $2.914370 \mathrm{E}-02$ & $3.367174 \mathrm{E}-02$ \\
\hline $6.500000 \mathrm{E}-01$ & $8.765479 \mathrm{E}-01$ & $9.091651 E-02$ & $1.020654 \mathrm{E}-01$ \\
\hline $7.000000 \mathrm{E}-01$ & $2.852176 \mathrm{E}-00$ & $3.529778 \mathrm{E}-01$ & $3.931476 \mathrm{E}=01$ \\
\hline $7.500000 E-01$ & $1.207867 \mathrm{E}+01$ & $5.169254 \mathrm{E}-00$ & $4.372553 \mathrm{E}-00$ \\
\hline $7.600000 E-01$ & $2.034901 \mathrm{E}+02$ & $1.125226 \mathrm{E}+01$ & $9.209500 \mathrm{E}-00$ \\
\hline $7.700000 \mathrm{E}-01$ & $1.781252 \mathrm{E}+04$ & $8.199627 \mathrm{E}+03$ & $2.480072 E+01$ \\
\hline $7.800000 \mathrm{E}-01$ & $2.004583 E+06$ & $4.127058 E+05$ & $1.340456 E+02$ \\
\hline $7.850000 \mathrm{E}-01$ & $4.851691 E+10$ & $3.581233 \mathrm{E}+08$ & $1.367465 E+02$ \\
\hline $7.900000 E-01$ & $2.115584 \mathrm{E} \cdot 09$ & $1.005519 \mathrm{E}+06$ & $9.598928 E+01$ \\
\hline $8.000000 \mathrm{E}-01$ & $8.178258 E+08$ & $9.232341 E+05$ & $2.671256 \mathrm{E}-00$ \\
\hline
\end{tabular}

Clearly, taking smaller step length greatly improves the results obtained.

As in table1, we have shown the performance of the new scheme (18b) when compared with implicit Euler scheme in table 2 for problem 2. Since trapezoidal scheme has the smallest error constant among linear multi-step methods of order 2 (Fatunla, 1988: 42-46), we have further decided to compare the performance of our scheme with it also since both require the same number of function evaluations per step and for the purpose of wider comparison. 
The results by both the trapezoidal method and our scheme are better than that by the backward Euler. The results by the trapezoidal scheme are slightly better than the ones by our scheme. As we approach the singularity point, our scheme behaves clearly well than both schemes. The ability of our scheme to quickly recover after the singularity point is clearly seen in the table.

\section{CONCLUSION}

A convergent and A-stable canonical backward differentiation formula has been derived for the solution of initial value problems of first ordinary differential equations with singular solution. The results obtained were quite encouraging when compared in particular with that by the conventional linear Euler backward differentiation formula although with an extra cost of computing square root. The ability of the scheme to continue fairly well after the singularity point is another advantage of the scheme over the linear Euler backward scheme.

\section{RLFERENCES}

Curtis, ('. I:, and Hirschfelder, J, O., 1952. Integration of Stiff Equations. National Academy of Sciences 38, 235 243

Dahlquisl. G., 1963. A Special Stability Problem for LMMs, BIT 3.27-43.

[uler, b.. 1913. Opera Ommia, Series Prima, 11, Leipzig and Berlin.

Fatumla. S. O., 1978. A Variable Order One Step Scheme for Numerical Solution of ODEs. Computer and Mathematics with Application 4:33-41.

Fatunla. S. O., 1988. Numerical Methods for IVPs in ODEs. Academic Press, San Dicgo.

Gear, C. W., 1969. The Automatic Integration of Stiff ODEs, in Information Processing 68 (A. J. H. Morrell, ed.). Amsterdam: North-Holland publishing co., 187 - 193.

Gear, C. W., 1971. Algorithm 407: DIFSUB for Solution of ODEs, Communications of ACM, 14. $185-190$.

Hall, G. and Watt, J. M. (eds), 1976. Moden Numerical Methods for ODL:s. Oxtord university press, London, pp. 116

Hindmarsh, A. C., 1974. GEAR: ODE System Solver, Revision 3, Report no. UCID - 30001, Lawrence Livermore lab; University of California, Livermore.

Hindmarsh, A. C., 1980. LSODE and LSODI: Two New Initial Value ODE solvers, ACM S[GNUM Newsletter 15, $10-11$.

Jain, M. K., 1987. Numerical Solution of Differental Equations. Wiley Lastem Limited, New Delhi, pp. 127.

Lambelt, J. D., 1973. Computational Methods in ODE. John Wiley and Sons, N.Y.

Scheid, I. J., 1989. Shamm's Outline of Theory and Problems of Numerical Analysis. McGraw-Hidl Companies, [ne. N.Y., pp. 220.

Taivo, O., and Onumanyi, P.. 1991. A Collocation Approximation of Singularly Perturbed Second Order O.D.E. Inter. J. Computer Mallss. 39: 205-211. 